\title{
Using the Lessons Learned From the Clinic to Improve the Preclinical Development of Antibody Drug Conjugates
}

\author{
Dowdy Jackson $•$ David Stover
}

Received: 30 May 2014 / Accepted: 29 September 2014 / Published online: 23 October 2014

(C) The Author(s) 20I4. This article is published with open access at Springerlink.com

\begin{abstract}
The treatment options for cancer patients include surgery, chemotherapeutics, radiation therapy, antibody therapy and various combinations of these therapies. The challenge with each therapy is finding the balance between maximizing the antitumor efficacy while minimizing the dose limiting toxicities. Antibodies, unlike small molecule chemotherapeutics, selectively bind to cell surface tumor antigens and can be used to deliver radionucleotides or small molecule chemotherapeutic drugs directly to the tumor. Advances in antibody engineering, linker chemistry and the identification of potent cytotoxic drugs led to the recent approval of two antibody drug conjugates to treat breast cancer and lymphoma patients. We will discuss how the observations from the clinical development of antibody drug conjugates can guide the preclinical development of the next generation of antibody drug conjugates.
\end{abstract}

KEY WORDS antibody drug conjugate · preclinical · site specific

$\begin{array}{ll}\text { ABBREVIATIONS } \\ \text { ALCL } & \text { Anaplastic large cell lymphoma } \\ \text { ADC } & \text { Antibody drug conjugate } \\ \text { ALL } & \text { Acute lymphoblastic leukemia } \\ \text { AML } & \text { Acute myeloid leukemia } \\ \text { CL2A } & \text { maleimido-[short PEG]-Lys-PABOCO-20-O-SN-38 } \\ \text { DAR } & \text { Drug to antibody ratio } \\ \text { DLBCL } & \text { Diffuse large B-cell lymphoma } \\ \text { GBM } & \text { Glioblastoma multiforme } \\ \text { KD } & \text { Kilodalton } \\ \text { MMAE } & \text { Monomethylauristatin E } \\ \text { MMAF } & \text { Monomethylauristatin F } \\ \text { ND } & \text { Not disclosed } \\ \text { NHL } & \text { Non-hodgkin lymphoma } \\ \text { NNAA } & \text { Non-natural amino acid }\end{array}$

D. Jackson $(\square) \cdot D$. Stover

Agensys, Inc, I 800 Stewart Street, Santa Monica, California 90404, USA

e-mail: djackson@agensys.com

\author{
NSCLC Non-small cell lung cancer \\ PBD Pyrrolobenzodiazepine \\ PDX Patient derived xenograft \\ PK/PD Pharmacokinetic/Pharmacodynamic \\ Q3W Every 3 weeks \\ RCC Renal cell cancer \\ SCC Squamous cell carcinoma \\ SCLC Small cell lung cancer \\ SMCC N-succinimidyl 4-(N-maleimidomethyl)cyclohexane- I \\ carboxylate \\ SN-30 7-ethyl-10-hydroxycamptothecin \\ SPDB N-succinimidyl-4-(2-pyridyldithio)butyrate \\ SPP N-succinimidyl 4-(2-pyridyldithio)pentanoate
}

\section{INTRODUCTION}

Small molecule chemotherapeutics and antibodies are commonly used to treat cancer patients. Small molecule chemotherapeutics, such as doxorubicin, paclitaxel and vinblastine, are delivered systemically, have relatively short serum halflives and do not specifically target tumor cells. These molecules not only kill rapidly dividing tumor cells but they can also kill some normal cells, which results in various dose limiting toxicities (DLTs), such as neutropenia. DLTs reduce the amount of drug that can be administered to a patient which can reduce the effectiveness of a drug.

Antibodies are also delivered systemically but tend to have long serum half-lives and selectively bind to tumor cells. Antibody drug conjugates (ADCs) are a combination of the small molecule chemotherapeutic and the antibody thus allowing the selective delivery of the small molecule chemotherapeutic drugs to tumor cells and not normal cells.

Although average Drug/Antibody Ratios (DAR) are typically in the range from 2 to 4 drugs per antibody, most ADCs in clinical development today are a mix of antibodies carrying 
0 to as many as 12 cytotoxic drugs covalently conjugated to specific amino acids on the antibody via a chemical linker. ADCs bind to cell surface antigens; they are typically internalized by the cells and processed by the lysosomes where the cytotoxic drugs are released into the cytotsol. Once the drugs are released from the lysosomes, they bind to their intracellular target, disrupt a critical cellular process and kill the cell.

The preclinical development of ADCs involves several steps which include the identification of the tumor antigen, the discovery and characterization of an antibody against the tumor antigen, the identification of the appropriate cytotoxic drug, the conjugation of the cytotoxic drug to the antibody and the characterization of the amount of aggregate and other physiochemical properties of the ADC. The preclinical evaluation of ADCs includes antibody/antigen binding studies, in vitro cytotoxic studies, in vivo anti-tumor efficacy studies, pharmacokinetic and the toxicology studies in rodent and non-human primates.

The observations from the clinical development of ADCs have been crucial in refining the preclinical development of ADCs. Improvements in antibody engineering, potency of cytotoxic drugs and improvements in the linker chemistry lead to the current generation of ADCs. We will discuss how data from the current clinical studies can be used to improve the preclinical development of the next generation of ADCs.

\section{ADCS: A HISTORICAL PERSPECTIVE}

Paul Ehrlich, the German physician and scientist, described the concept of delivering a toxophore, a cytotoxic drug, selectively to tumors. ADCs are the embodiment of this concept. The first generation of ADCs used common chemotherapeutic drugs such as methotrexate, vinblastine and doxorubicin as cytotoxic drug payloads. KS1/4 and BR96 were the first antibodies to enter clinical development as ADCs.

$\mathrm{KS} 1 / 4$ was a murine IgG2a antibody against a 40 and $42 \mathrm{kD}$ glycoprotein expressed by the human lung adenocarcinoma cell line, UCLA-P3 (1). The KS1/4 antigen is expressed by several cancers including ovarian, lung, pancreatic and colorectal cancers. KS1/4 was conjugated to methotrexate (KS1/4-methotrexate) or vinblastine (KS1/4DAVLB) $(2,3)$. There were 6 molecules of methotrexate and 4 to 6 molecules of vinblastine per antibody on lysines using hemisuccinate linkers. Preclinical in vivo anti-tumor efficacy was reported for the $\mathrm{KS} 1 / 4$-methotrexate and the $\mathrm{KS} 1 / 4$ DAVLB ADCs but no significant clinical responses were observed. Patients treated with the $\mathrm{KS} 1 / 4$ antibody or $\mathrm{KS} 1 / 4$ ADCs produced an antibody response against the mouse antibody, also known as a human anti mouse antibody (HAMA) response. Although the HAMA response has been reported to result in rapid systemic clearance of the antibody thus rendering the antibody or in this case ADC ineffective, high serum levels of the $\mathrm{KS} 1 / 4$ antibody were reported in patients treated with the higher doses of the KS1/4 antibody or ADCs. Subsequent ADCs used chimeric, humanized or fully human antibodies to reduce the patient's immune response against the antibody.

BR96-Doxorubicin (SGN-15) was licensed by Seattle Genetics from Bristol-Meyer Squibb (BMS) (4). SGN-15 was a chimeric antibody against the Lewis Y (CD174) antigen that was conjugated to doxorubicin (adriamycin) using an acid labile, 6-maleimidocaproyl hydrazone linker $(5,6)$. In preclinical studies, SGN-15 was able to selectively kill Lewis Y expressing cells in both in-vitro cytotoxicity and in in-vivo tumor efficacy studies yet it was unable to show statistically significant clinical benefit and further development was discontinued. The lack of clinical benefit has been attributed to several factors including the insufficient cytotoxic potency of doxorubicin, the instability of the hydrazone linker and the expression of Lewis Y by several normal tissues. (7-9).

CMD-193, which was developed by Wyeth Pharmaceuticals, Inc, was a humanized antibody (hu3S193) against the Lewis $\mathrm{Y}$ antigen that was conjugated to the DNA synthesis inhibitor, $\mathrm{N}$-acetyl gamma calicheamicin dimethyl hydrazide (Calicheamicin) using the acid labile 4-(4'-acetylphenoxy) butanoic acid) linker (10). In preclinical studies, CMD-193, like SGN-15, was able to kill Lewis Y expressing tumors in both in vitro cytotoxicity studies and in vivo tumor efficacy studies (10). In a phase I clinical study, myelosuppression and prolonged liver uptake which affected liver function were the most significant adverse events (11). Further clinical development of CMD-193 was terminated.

Gemtuzumab Ozogamacin (Mylotarg), which was also developed by Wyeth Pharmaceuticals, Inc (now Pfizer) and Celltech (now a part of UCB Brussels), was a humanized antiCD33 IgG4 antibody, conjugated to Calicheamicin via the acid labile 4-4'-acetylphenoxy butanoic acid linker. Mylotarg had an average of 3 molecules of Calicheamicin per antibody and approximately $50 \%$ of the antibody did not contain Calicheamicin (12). Despite the high amount of unconjugated antibody, which could reduce its effectiveness, Mylotarg was able to kill CD33 expressing cells in in-vitro cytotoxicity studies and in preclinical in-vivo tumor efficacy studies (13). Mylotarg showed promise in clinical studies and was the first ADC to gain approval from the food and drug administration (FDA) in May 2000. Despite the promising clinical data, Mylotarg was eventually withdrawn from the market, in 2010, due to a lack of efficacy and an increased number of deaths in acute myelogenous leukemia (AML) patients as compared to patients treated with the standard of care chemotherapeutics (14).

Subsequent preclinical efforts to develop ADCs focused on the identification of potent cytotoxic drugs, linker chemistries 
that provide sufficient in vitro and in viwo stability and refinement of conjugation conditions to reduce the amount of unconjugated antibody.

In 2011 Adcetris (Brentuximab vedotin) was approved by the FDA to treat systemic anaplastic large cell lymphoma (sALCL) and Hodgkin's lymphoma (HL). Adcetris is a chimeric IgG1 antibody against CD30 that is conjugated to an average of four molecules of the cytotoxic drug, monomethyl auristatin $\mathrm{E}$ (MMAE), via the thiols on cysteines using a valine-citrulline dipeptide linker with a p-aminobenzyl alcohol (PABA) spacer. MMAE is a potent inhibitor of cell growth in vitro. MMAE's average in vitro $\mathrm{IC}_{50}$ values, against a panel of human tumor cell lines, were commonly below $1 \mathrm{nM}$ as opposed to doxorubicin which had an average $\mathrm{IC}_{50}$ value of $631 \mathrm{nM}(15)$.

In 2013, Kadcyla (T-DM1) was approved by the FDA to treat Her2 expressing metastatic breast cancer patients. Kadcyla is a humanized IgG1 antibody against Her2 that is conjugated to an average of 3.5 molecules of the cytotoxic maytansine drug, N2'-deacetyl-N2'-(3-mercapto-1oxopropyl)-maytansine (DM1), via the amines on lysines using the 4-[N-maleimidomethyl] cyclohexane-1carboxylate (MCC) linker. DMl's average in vitro cytotoxicity $\mathrm{IC}_{50}$ values against several tumor cell lines were less than $1 \mathrm{nM}(16,17)$.

The clinical development of ADCs is expanding rapidly. Over $30 \mathrm{ADC}$ s are in clinical development (Table I). The majority of ADCs are in either Phase I, Phase II or have active clinical efforts in both Phase I and Phase II (Fig. 1). Only one ADG, Inotuzumab Ozogamicin, has an active Phase III clinical effort. Over 15 ADCs have been withdrawn from clinical development for various reasons, which include a lack of antitumor response, unacceptable toxicity or a change in company strategy. The results from the clinical development of ADCs influence the preclinical development efforts, which lead to the creation of the next generation of ADCs.

There are several key areas of focus for the preclinical development of ADCs. These areas include the selection of an ADC target, the selection of an appropriate antibody and the selection of appropriate cytotoxic drugs and linkers. Furthermore, attention has to be given to characterizing the physiochemical properties of the ADCs, evaluation of the in vitro cytotoxicity, the in vivo anti- tumor efficacy of the ADCs, the pharmacokinetic properties of the ADCs and their toxicology/safety profiles (Fig. 2).

\section{TARGET SELECTION}

The optimal selection criteria for cell surface tumor antigens are that the antigen should be expressed selectively and at higher levels by tumors than normal tissues and the antigens are internalized by the tumor cell. Furthermore it would be preferred if the tumor antigens are expressed homogeneously to increase the number of cells killed by the ADC. It is unclear what the threshold receptor density or the internalization rate should be for a tumor antigen because the receptor density and the internalization rates may vary depending on the tumor antigen and tumor type. Thus far three ADGs have been approved to treat cancer patients. Those ADCs are Gemtuzumab Ozogamicin (Mylotarg), which targets CD33 expressing tumors; Adcetris, which targets CD30 expressing tumors and Kadcyla, which targets Her2 expressing tumors (Table II).

\section{CD33}

CD33 (Siglec-3) is expressed by the precursor myeloid and monocytic cells. CD33 is expressed in approximately 85-90\% of adult and child AML patients and in $100 \%$ of acute promyelocytic leukemia (APL), which is a subset of AML $(18,19)$. CD33 expression correlates with poor prognosis for children with ALL (20). High levels of CD33 expression have been reported in the bone marrow of AML patients while substantially lower levels of expression have been observed in normal bone marrow (21). The restricted expression of CD33 in normal tissues and the high levels of expression in AML made CD33 an attractive therapeutic target.

Three ADCs, Mylotarg, AVE9633, and SGN-CD33A, have been developed to treat patients with CD33 expressing tumors.

AVE9633 was a humanized IgG1 antibody conjugated to an average of 3-5 molecules of N2'-deacetyl-N2'-(4mercapto-4-methyl-1-oxopentyl)-6-methylmaytansine (DM4) via a hindered disulfide linker on lysines. In preclinical studies, AVE9633 selectively killed AML cell lines and AML patient samples in vitro $(22,23)$. Three phase I studies were performed and a total of 54 AML patients were treated with AVE9633. Patients were treated on day 1 of a 21 day cycle (Day 1), days 1 and 8 (Day 1/8) and days 1,4 and 7 (Dayl/4/7) of a 28 day cycle. The day 1 and day $1 / 4 / 7$ schedules were terminated early due lack of cytotoxic activity at doses higher than the doses that saturated binding to CD33 (22).

Mylotarg, as was previously mentioned, was removed from the market in 2010, which leaves SGN-CD33A as the only ADC currently in clinical development against CD33. SGNCD33A is composed of humanized IgG1 antibody (h2H12), which contains two engineered cysteines at position 239 (S239C) on the antibody heavy chains. The DNA synthesis inhibitor, made by the former Spirogen LTD (now AstraZeneca/MedImmune), pyrrolobenzodiazepine (PBD), is covalently conjugated to each site specific cysteine using the 
Table I ADCs in Clinical Development

\begin{tabular}{|c|c|c|c|c|c|}
\hline ADC & Target & Phase of Development & Indication & Isotype & Payload \\
\hline ABT-4I4 & Activated EGFR/EGFRvIII & Phase I & GBM, SCC & $\lg G \mid$ & MMAF \\
\hline AGSI6M8F & ENPP3 & Phase I & $\mathrm{RCC}$ & $\lg G 2$ & MMAF \\
\hline ASG22M6E & Nectin 4 & Phase I & Solid tumors & $\lg G \mid$ & MMAE \\
\hline AGS67E & CD37 & Phase I & NHL, CLL, AML & $\lg G 2$ & MMAE \\
\hline AMG 172 & CD27L & Phase I & RCC & $\lg G \mid$ & DMI \\
\hline AMG-595 & EGFRvIII & Phase I & GBM & ND & DMI \\
\hline ASGI5E & SLITRK6 & Phase I & Solid tumors & $\lg G 2$ & MMAE \\
\hline Bay $94-9343$ & Mesothelin & Phase I & Solid tumors & $\lg G \mid$ & DM4 \\
\hline BT-062 & CDI38 & Phase I/II & Multiple myeloma & $\lg G 4$ & DM4 \\
\hline Glembatumumab Vedotin (CDX-0I I) & GPNMB & Phase II & Breast cancer & $\lg G 2$ & MMAE \\
\hline IMGN-853 & FOLRI & Phase I & Solid tumors & $\lg G \mid$ & DM4 \\
\hline IMMU- 130 & CEACAM5 & Phase I & Colorectal & $\lg G \mid$ & $\mathrm{SN}-38$ \\
\hline IMMU-132 & TACSTD2 (TROP2) & Phase I & Epithelial cancer & $\lg G \mid$ & $\mathrm{SN}-38$ \\
\hline IMGN529 & CD37 & Phase I & $\mathrm{NHL}, \mathrm{CLL}$ & $\lg G \mid$ & DMI \\
\hline Inotuzumab Ozogamicin (CMC-544) & CD22 & Phase III & ALL & $\lg G 4$ & CalichDMH \\
\hline MDX-1203 & CD70 (MDX-। I I5) & Phase I & Clear cell RCC, B-NHL & ND & CC-1065 \\
\hline Milatuzumab & CD74 & Phase I/II & Multiple myeloma & $\lg G \mid$ & Doxorubicin \\
\hline MLN0264 & Guanylyl cyclase C & Phase I & Colorectal & ND & MMAE \\
\hline PF-0626350 & $5 T 4$ & Phase I & Solid tumor & ND & MMAF \\
\hline PSMA (BrUOG 263) & PSMA & Phase I/II & Prostate & $\lg G \mid$ & MMAE \\
\hline RG7450 (DSTP3086S) & STEAPI & Phase I & Prostate & ND & MMAE \\
\hline RG7458 (DMUC5754A) & MUCI6 & Phase I & Ovarian & $\lg G \mid$ & MMAE \\
\hline RG7593 (DCDT2980S) & CD22 & Phase I & $\mathrm{NHL}$ & $\lg G \mid$ & MMAE \\
\hline RG7596 (DCDS450 IA) & CD79b & Phase I/II & $\mathrm{NHL}$ & $\lg G \mid$ & MMAE \\
\hline RG7598 & ND & Phase I & ND & ND & Undisclosed \\
\hline RG7599 (DNIB0600A) & $\mathrm{NaPi} 2 \mathrm{~b}$ & Phase I & Ovarian, NSCLC & $\lg G \mid$ & MMAE \\
\hline RG7600 & ND & Phase I & ND & ND & ND \\
\hline RG7636 & Endothelin B receptor & Phase I & Melanoma & ND & MMAE \\
\hline SAR34I9 & CDI9 & Phase I & DLBCL, ALL & $\lg G \mid$ & DM4 \\
\hline SAR566658 & CA6 & Phase I & Solid tumor & $\lg G \mid$ & DM4 \\
\hline SCI6LD6.5 & Fyn3 & Phase I/II & SCLC & ND & D6.5 \\
\hline SGN-CDI9A & CDI9 & Phase I & $\mathrm{ALL}, \mathrm{NHL}$ & ND & MMAF \\
\hline SGN-CD33A & CD33 & Phase I & AML & $\lg G \mid$ & PBD (SGD- | 882) \\
\hline SGN-LIVIA & LIV-I & Phase I & Breast cancer & ND & MMAE \\
\hline
\end{tabular}

ND not disclosed, SN-30 7-ethyl- I0-hydroxycamptothecin, PBD Pyrrolobenzodiazepine, MMAE Monomethylauristatin E, MMAF Monomethylauristatin F, GBM Glioblastoma multiforme, RCC Renal cell cancer, SCLC Small cell lung cancer, NSCLC Non-small cell lung cancer, AML Acute Lymphoblastic Leukemia, NHL Nonhodgkin lymphoma, ALL Acute lymphoblastic leukemia, DLBCL Diffuse large B-cell lymphoma, SCC Squamous cell carcinoma

maleimidocaproyl-valine-alanine linker. SGN-CD33A is the first publically disclosed site specific ADC in clinical development.

\section{CD30}

CD30 (TNFRSF8) is expressed on activated T and B cells and a small population of eosinophils. It is also expressed in sALCL, HL, mature $\mathrm{T}$ cell lymphomas and B cell derived non-Hodgkins lymphoma (NHL). sALCL is a rare type of
NHL, comprising approximately $3 \%$ of all NHL but is one of the more common $\mathrm{T}$ cell lymphomas (24). CD30 is expressed uniformly on sALCL (25). A soluble form of CD30 has been reported in the sera of cancer patients but this does not seem to have a deleterious effect on Adcetris (26). Limited expression of CD30 has been reported in normal tissues and high expression has been reported in sALCL, HL and other malignancies (27).

Adcetris had objective response rates of $75 \%$ for Hodgkin's lymphoma $(\mathrm{HL})$ patients and $86 \%$ for sALCL patients. 


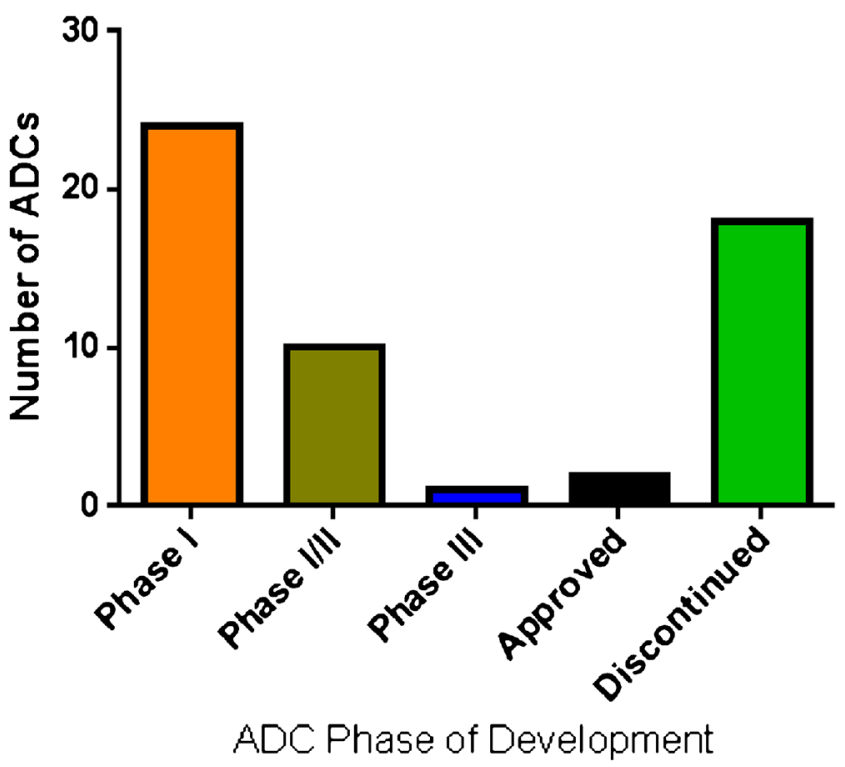

Fig. I Clinical development of ADCs.

Tumor reductions were reported in $94 \%$ of the HL patients and $97 \%$ of the sALCL patients (28). Interestingly CD30 expression in diffuse large B cell lymphoma (DLBCL) patients did not correlate with patient response to Adcetris. Tumor responses were observed in DLBCL patients that had undetectable expression of CD30 via immunohistochemical evaluation (29). These data suggest that CD30 expression alone in
DLBCL patients may not predict response to Adcetris. It also suggests that low CD30 expression is sufficient for responses to Adcetris.

\section{Her2 (ERBB2)}

Unlike CD33 and CD30, which are predominately expressed by hematological malignancies, Her2 is predominantly expressed by solid tumors. In particular, it is expressed in breast, gastric and ovarian cancer amongst others. Her2 is over expressed by approximately $25 \%$ of patients with invasive breast cancer. It is uniformly expressed in breast cancer and high expression of Her2 correlates with poor prognosis (30-33). Her 2 is also expressed by several normal tissues including, the skin, respiratory tract, heart, gastrointestinal epithelial cells and in the urinary and reproductive tracts (34). Kadcyla, also known as T-DM1, was approved in 2013 for Her2 expressing late stage breast cancer patients. The objective response rate in the pivotal EMILIA trial was $43.6 \%$ for patients treated with Kadcyla and 30.8\% for patients treated with lapatinib and capecitabine (35).

\section{Solid Tumor Antigens}

Developing antibody therapeutics for targets expressed in solid tumors may offer some additional challenges that may

\section{a Creating the ADC}

\begin{tabular}{|c|c|c|c|}
\hline Target ID & Antibody Generation & Antibody Characterization & Conjugation \\
\hline $\begin{array}{l}\text { - High and homogeneous } \\
\text { expression in the majority } \\
\text { of patient tumors. } \\
\text { - Low expression in normal } \\
\text { tissues. } \\
\text { - Target is internalized. }\end{array}$ & $\begin{array}{l}\text { - Use mice or phage display } \\
\text { methods to generate } \\
\text { antibodies. } \\
\text { - Generate sufficient } \\
\text { quantities of antibody. }\end{array}$ & $\begin{array}{l}\text { Binds with high affinity to } \\
\text { the human antigen. } \\
\text { - Specific for the antigen. } \\
\text { to rodent and norhuman } \\
\text { primate versions of the } \\
\text { antigen. }\end{array}$ & $\begin{array}{l}\text { - Optimization of conditions } \\
\text { - Low aggregation. } \\
\text { - High monomeric content. } \\
\text { - Consistent DAR. } \\
\text { - High yield. } \\
\text { - No loss of binding affinity. }\end{array}$ \\
\hline
\end{tabular}

- No sequence abnormalities.

\section{b Testing the ADC}

\section{In vitro cytotoxicity}

- Kills antigen expressing cells. (low nM-pM IC $\mathrm{I}_{50}$ values)

- Does not kill norantigen expressing cells.

- Kills cells with known drug resistance mechanisms.

\section{payload in serum (in vitro)}

- Has minimal release of the payload in the serum of rodents (tumor bearing and non tumor bearing).

- Has minimal release of the payload in the serum of non-human primates.

- Acceptable pharmacokinetic properties (i.e. $\left.\mathrm{T}_{1 / 2}, \mathrm{AUC}\right)$

\section{In vivo Tumor Efficacy}

- Induces tumor stasis or regression in multiple human tumor models. (Cell line xenografts, PDX, GEMMs)

- No obvious signs of toxicity at efficacious exposures.

- Not efficacious in non antigen expressing tumors.

\section{In vivo Toxicology}

- Identify the MTD and DLTs in rodents and norhuman primates.

- Determine the serum exposures where DLTs were observed. (Toxicokinetics)

- Histological analysis of tissues.

- Analysis of blood chemistry.

- Is the toxicity reversible, manageable and can it be monitored?

Fig. 2 Preclinical development and evaluation of ADCs. 
Table II List of Approved ADCs

\begin{tabular}{llllll}
\hline ADC & Target & Isotype & Payload & Indication & Date of Approval \\
\hline Mylotarg & CD33 & $\operatorname{lgG} 4$ & Calicheamicin & AML & $2000\left(\right.$ US) ${ }^{\text {a }}$ \\
Kadcyla (T-DMI) & Her2 & $\operatorname{lgG}$ & DMI & Her2 positive late stage breast & 20 I3 (US) \\
Adcetris (SGN-35) & CD30 & $\operatorname{lgG}$ & MMAE & SALCL, relapsed/refractory Hodgkin lymphoma & 20। I (US) \\
\hline
\end{tabular}

AML Acute Lymphoblastic Leukemia, ACLC Anaplastic large cell lymphoma

a Withdrawn in 2010

not exist for hematological cancer targets. For hematological cancers, it is possible to determine the dose that achieves receptor saturation. Once that dose is achieved, the addition of higher amounts of ADC would not be beneficial. Antibody delivery to solid tumors, however, is very complex. Solid tumors have high intratumoral pressures, due to the lack of lymphatics (36). Solid tumors have leaky blood vessels and chaotic blood flow, which can result in heterogeneous distribution of the antibody within the tumor thus rendering portions of the tumor inaccessible to the antibody or ADC (36-38). Another factor to consider is the large size of antibodies, which have a molecular weight of $\sim 150 \mathrm{kD}$. The large size of the antibodies may limit their ability to efficiently penetrate deep within a solid tumor mass thus leaving portions of the tumor untreated and free to continue to grow (39). In addition to the physical properties of the antibody, the expression of the antigen must also be taken into consideration. If the tumor antigen is heterogeneously expressed within the tumor, portions of the tumor could be devoid of ADC thus allowing portions of the tumor to be untreated, which enables tumor growth. Lastly antibody affinities may impact anti-tumor efficacy of an ADC. Antibodies with high affinities are tightly bound to the antigen and may be unable to penetrate the tumor as well as antibodies with lower affinities, which will reduce the effectiveness of the $\mathrm{ADC}(40,41)$.

The current operating hypothesis is an ADC must be internalized in order for the drug to be released from the antibody and subsequently kill the antigen expressing cell. This has resulted in linkers that are designed to release their drug in a low $\mathrm{pH}$ environment or upon proteolysis using lysosomal enzymes. Although this is one way ADCs function, it is possible that some ADC drugs could be released without being internalized. The anti-CEACAM5 ADC (Epratuzumab-SN-38), which uses the CL2A linker combined with the SN-38 drug, has been reported to deliver the ADC to the antigen expressing tumors in vivo but releases the SN-38 drug without being efficiently internalized (42). The authors state that $\mathrm{SN}-38$ is slowly released from the anti-CEACAM5 antibody (@50\%/day) and the in vitro internalization rate for the anti-CEACAM5 antibody is slow. However, it is not clear that in vitro rates of internalization necessarily predict in vivo rates. Thus, the use of $\mathrm{pH}$ sensitive dyes (i.e. $\mathrm{pHRodo}^{\mathrm{TM}}$ ) or far red dyes to label ADCs to evaluate the internalization and intracellular trafficking of ADCs in vivo, could be a useful tool to determine the importance of internalization to in vivo efficacy.

\section{SELECTING THE ANTIBODY}

\section{Antibody Isotype}

The first ADCs were developed using murine antibodies. Patients developed HAMA responses against the murine antibody, which may have limited the effectiveness of these ADCs (3). The majority of the ADCs currently in clinical development are chimeric, fully human or humanized IgG1 antibodies. IgG2 and IgG4 isotypes are also being developed as ADCs (Table I).

There are structural differences between the various antibody isoforms (Fig. 3). The IgG2 isotype has four interchain disulfide bonds in the hinge region while the $\mathrm{IgGl}$ and $\mathrm{IgG} 4$ isoforms have two interchain disulfide bonds (Fig. 3). The conjugation of the cytotoxic drug to the antibody, on cysteines, requires a partial reduction of the disulfides in the hinge region of the antibody. Tris (2-carboxyethyl)-phosphine (TCEP) is commonly used to reduce the antibody thus increasing the number of free thiols available for conjugation. IgG2 antibodies require higher concentrations of TCEP and longer reaction times than is required for IgG1 antibodies (43). Furthermore the IgG2 isoform exists as an A form, a B form and a hybrid A/B form (44). The significance of how these different IgG2 isoforms affect the production or conjugation of a drug to an ADC is unclear.

Some IgG1 antibodies have antibody-dependent cellular cytotoxicity (ADCG) and complement dependent cytotoxicity (CDC), which is achieved through binding of the antibody's $\mathrm{Fc}_{\mathrm{C}}$ domain to the $\mathrm{Fc} \gamma$ receptors on natural killer cells, monocytes and macrophages (45). IgG2 and IgG4 antibodies have little to no CDC activity (46). Although IgG1 antibodies, such as Trastuzumab, can have ADCG and CDG activities, it is unclear whether these activities are important for an ADC (47). To address the potential differences between IgG1, IgG2 and IgG4 ADCs, an anti-CD70 ADC, which was conjugated to valine-citrulline monomethyl auristatin $\mathrm{F}$ (MMAF) (43). 

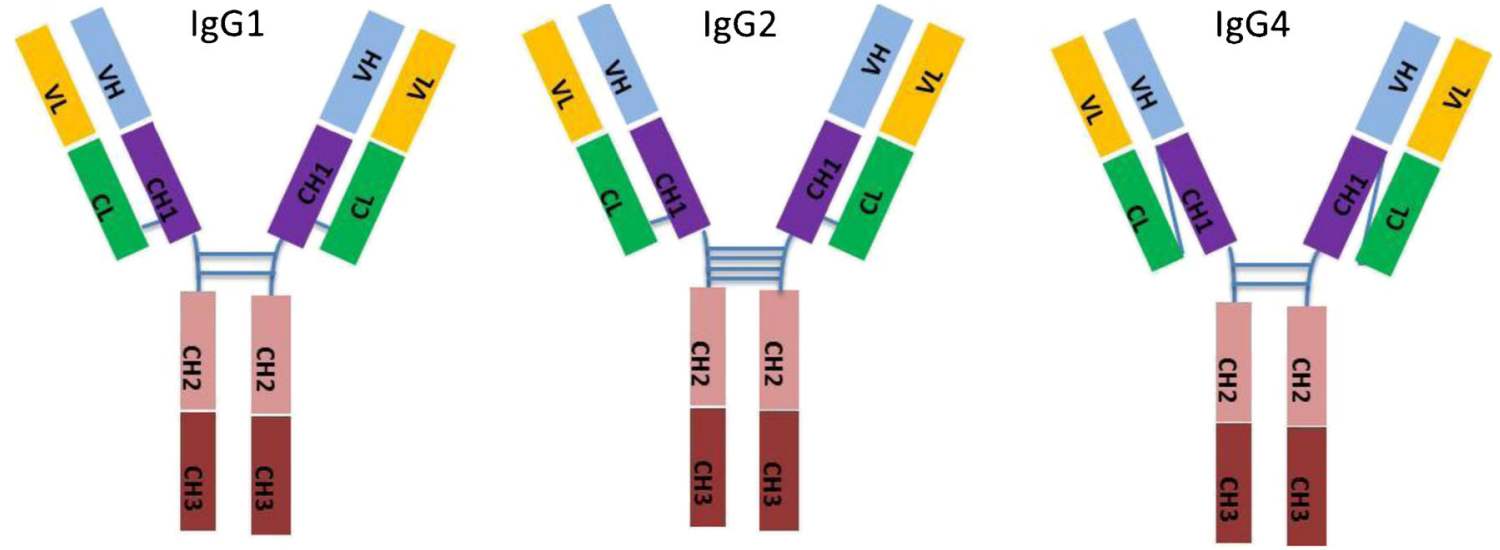

Fig. 3 lgG isotypes used as ADCs.

The authors also mutated the $\mathrm{FC}_{\mathrm{C}}$ domains of the anti-CD70 IgG1 (IgGlvl) and IgG4 (IgG4vl) ADCs to reduce binding affinity to the Fc $\gamma$ receptors. The IgGlvl-vc4 ADC had better in viwo tumor efficacy than the native IgG1-vcF4 ADC, which was attributed to increased serum exposure of the Fc mutated IgG1 ADC. This provided only a partial explanation for the enhanced efficacy of the Fc mutated IgG1 ADC because the wild type IgG2-vcF4 ADC had comparable serum exposure as the Fc mutated IgG1 ADC yet it was not as efficacious as the mutated IgG1 ADC.

\section{CYTOTOXIC DRUGS AND LINKERS}

\section{Cytotoxic Drugs}

The drugs used for ADCs inhibit cellular processes that are vital for cell proliferation and/or survival, such as tubulin polymerization or DNA replication (Table III). Inhibition of tubulin function or DNA synthesis induces apoptotic cell death. The current cytotoxic drugs, used as ADG payloads, are typically 100-2,000 fold more potent than doxorubicin, vinca alkaloids or taxanes (48-51). Although the majority of drugs in clinical development inhibit tubulin function or DNA synthesis, new drugs which inhibit critical cellular processes, such as RNA synthesis or membrane disruption are being developed as ADC payloads and may enter into clinical development. The cytotoxic drugs or their metabolites have varying degrees of membrane permeability. MMAE, for example, is membrane permeable and when released from a cell it may kill neighboring cells by a process known as bystander effect (52). MMAF has reduced membrane permeability and the metabolite, Cys-mcMMAF, does not exhibit bystander activity. Bystander activity may be useful in a solid tumor setting when the target antigen is heterogeneous expressed in the tumor. An anti-mesothelin ADC, Anetumab ravtansine, which is a fully human anti-mesothelin antibody conjugated to an average of 3.2 molecules of the cytotoxic maytansine drug DM4, was reported to exhibit bystander activity in tumor xenograft models (53). Tumor regressions were observed in tumor models where only $20 \%$ of the cells expressed mesothelin, which suggests that the non-mesothelin cells were killed by the release of the DM4 metabolite from the mesothelin expressing cells.

\section{Linkers}

Linkers are grouped into two classes (Table IV). They are either cleavable or non-cleavable. Cleavable linkers can be dipeptide linkers, such as valine-citrulline, and are cleaved by proteases (i.e. Cathepsin B), which results in the release of the cytotoxic drug. Cleavable linkers can also be acid labile, such as hydrazones and hindered disulfides, where the lysosomal environment results in the release of the cytotoxic drug. ADCs with non-cleavable linkers are degraded in the lysosome, which results in the formation of an amino acid-drug combination (i.e. Cys-mcMMAF). Although the linkers can be processed by the enzymes or environment of the lysosome, some linkers such as maleimide linkers, can transfer the cytotoxic drug to free sulfhydryls such as the cysteine on human serum albumin (HSA) or glutathione via a retro-michael reaction $(54,55)$. This can cause a $25 \%$ or greater decrease in the tumor exposure of the ADC (54). This decrease in tumor exposure may reduce the effectiveness of the ADC.

The linker/drug combination can influence the pharmacokinetics and the toxicology profile of the ADC. A humanized antibody (huC242) against CanAg/MUC1 was conjugated to the SPP-DM1 and SPDB-DM4 linker/drug combinations and evaluated in preclinical and clinical studies (Table V). The serum half-life in humans for the huC242SPP-DM1 (Cantuzumab Mertansine), using a Q3W dosing schedule, ranged between 18 and 48.5 hrs while the serum half-life for huC242-SPDB-DM4 (IMGN242) was between 60 and 120 hrs (56). In addition to the apparent differences in the half-lives of the ADCs, the dose limiting toxicities (DLT) 
Table III ADC Payloads

\begin{tabular}{lll}
\hline Drug/Payload (Payload name) & Mechanism of action & Stage of development \\
\hline Auristatin (MMAE/MMMAF) & Tubulin assembly inhibitor & Approved/Clinical \\
Maytansinoid (DMI/DM4) & Tubulin assembly inhibitor & Approved/Clinical \\
Tubulysin & Tubulin assembly Inhibitor & Preclinical \\
SN-38 & Topoisomerase inhibitor & Clinical \\
Calicheamicin & DNA synthesis inhibitor & Clinical \\
Doxorubicin & DNA synthesis inhibitor & Clinical \\
Pyrrolobenzodiazepine (SGD-1882) & DNA synthesis inhibitor & Clinical \\
Duocarmycin (CCI065) & DNA synthesis inhibitor & Clinical \\
D6.5 & DNA synthesis inhibitor & Clinical \\
a- Amanitin & RNA polymerase II inhibitor & Preclinical \\
\hline
\end{tabular}

were also different. The DLT for the huC242-SPP-DM1 $\mathrm{ADC}$ was elevated hepatic transaminase and the DLT for the huC242-SPDB-DM4 ADG was ocular toxicity, which was only observed in patients with low serum levels of CanAg (56). These data suggest that the different drug linker combinations can have significant effects on the pharmacokinetics of the $\mathrm{ADC}$ and the toxicology profiles. Interestingly, several ADCs have been reported to have reversible ocular toxicity in patients (Table VI). Ocular toxicity has been observed primarily for ADCs conjugated to DM4 or MMAF but Kadcyla, which is conjugated to DM1, reported ocular toxicity in $31.3 \%$ of the patients in a phase II study (57). These observations have resulted in the inclusion of ophthalmologic evaluation in preclinical toxicology studies. A patent application, US 20120282282 Al, was filed by Immunogen where the claims suggest that the ocular toxicity for DM4 ADCs can be decreased by modifying the charge of the linker. The uncharged SPDB linker was reported to display decreased ocular toxicity in a rabbit model than the charged sulfo-SPDB linker.

\section{Drug Resistance}

One of the reasons why cancer therapies fail is due to drug resistance (58). There are several mechanisms responsible for resistance to chemotherapies, which includes the expression of drug-efflux pumps (59). Drug-efflux pumps lower the intracellular concentration of the drug which renders the drug ineffective. The expression of several drug efflux pumps including multi-drug resistance $1(\mathrm{MDR} 1 / \mathrm{ABCB} 1)$, multi-drug resistance protein 1(MRP1/ABCG1) and breast cancer resistance protein (BCRP/ABCG2) have been implicated in resistance to various chemotherapeutics. Several cytotoxic drugs, used for ADCs, such as MMAE, DM1 and Calicheamicin, are known substrates of the drug efflux pump MDR1 (ABCB1) (60,61). MDR1 substrates tend to be hydrophobic drugs therefore modifying the metabolites to be polar or negatively charged may reduce the drugs from being pumped out of the cells via MDR1 (62).To counteract the MDR1 drug efflux pump, the linker used to conjugate DM1 to an anti-EpCam antibody was changed from N-succinimidyl4-(maleimidomethyl) cyclohexane-1-carboxylate (SMCC) to the hydrophobic linker $\mathrm{PEG}_{4} \mathrm{Mal}$. The resulting metabolite, lysine-PEG4Mal-DM1 was able to kill MDR1 expressing cells while the lysine-SMCG-DM1 was unable to kill MDR1 expressing cells (63). These data suggest that selection of the appropriate linker may be important to avoid resistance mediated via drug efflux pumps. In addition to selecting the appropriate linker to avoid drug efflux pumps, cytotoxic drugs

Table IV Linkers Used for ADCs

\begin{tabular}{llll}
\hline Linker name & Cleavable/Non-cleavable & Conditions for payload release & Payload \\
\hline Valine-citrulline $(\mathrm{vc})$ & Cleavable & Protease & MMAE \\
SPP & Cleavable & Reducing/Oxidizing environment & DMI \\
SPDB & Cleavable & Reducing/Oxidizing environment & DM4 \\
6-maleimidocaproyl hydrazide & Cleavable & Acid labile & Doxorubicin \\
4-(4'-acetylphenoxy) butanoic acid) & Cleavable & Acid labile & Calicheamicin \\
CL2A & Cleavable & PH mediated & SN-38 \\
SMCC & Non-cleavable & Antibody degradation & DMI \\
Maleimidocaproyl $(\mathrm{mc})$ & Non-cleavable & Antibody degradation & MMAF \\
\hline
\end{tabular}

SPP N-succinimidyl 4-(2-pyridyldithio)pentanoate, SMCC N-succinimidyl 4-(N-maleimidomethyl)cyclohexane-I carboxylate, SPDB N-succinimidyl4-(2-pyridyldithio)butyrate, CL2A maleimido-[short PEG]-Lys-PABOCO-20-O-SN-38 
Table V Clinical Summary of huC242 ADCs

\begin{tabular}{llllllll}
\hline Antibody & Payload & Linker & Schedule & MTD $\left(\mathrm{mg} / \mathrm{m}^{2}\right)$ & $T_{1 / 2}$ (hours) & DLT & Clinical Phase \\
\hline huC242 & DMI & SPP & QIW & II5 & $13-4 \mid$ & Elevated hepatic transaminase & I \\
huC242 & DMI & SPP & Q3W & 235 & $18-48.5$ & Elevated hepatic transaminase & I \\
huC242 & DM4 & SPDB & Q3W & 168 & $60-120$ & Reversible ocular toxicity \\
huC242 & DM4 & SPDB & Q3W & 168 & N/A & Reversible ocular toxicity \\
\hline
\end{tabular}

that are not substrates for efflux pumps, such as PBDs, may also be considered. SGN-CD33A is conjugated to the PBD,SGD-1882, which is not an MDR1 substrate. SGNCD33A can kill MDR1 expressing CD33 positive AML patient samples and is currently in Phase I.

Several methods, using fluorescent substrates for the various drug efflux pumps, have been used to assess the activity of the pumps and may be useful in characterizing the contributions of the various pumps to resistance to the ADC metabolites (64). Rhodamine 123, for example, can be used to assess drug efflux pump activity in cell lines and specific inhibitors of various efflux pumps can be used to determine the specific drug efflux pump's contribution to the efflux activity in a cell line or patient derived cells (65).

\section{ANTIBODY CONJUGATION OPTIONS}

There are two methods used to covalently attach a cytotoxic drug to an antibody. Currently, the more conventional method, conjugates the cytotoxic drugs to an antibody's solvent exposed cysteine or lysine residues. The site specific conjugation method conjugates the cytotoxic drugs to natural or nonnatural amino acids that have been engineered at different locations on the antibody or to glycosyl moieties.

\section{Conventional Conjugation}

The majority of the ADGs in clinical development are in this category. The cytotoxic drugs are conjugated to antibodies via

Table VI ADCs with Ocular Toxicity in the Clinic

\begin{tabular}{llll}
\hline Antibody & Target & Linker & Payload \\
\hline SAR34I9 & CDI9 & SPDB & DM4 \\
IMGN853 & Folate Receptor a & SPDB & DM4 \\
SGN-75 & CD70 & mc & MMAF \\
SAR566658 & CA6 & SPDB & DM4 \\
BT-062 & CDI38 & SPDB & DM4 \\
huC242-DM4 & CanAg & SPDB & DM4 \\
SGN-CDI9A & CDI9 & mc & MMAF \\
Kadcyla & Her2 & SMCC & DMI \\
\hline
\end{tabular}

the thiols on solvent exposed cysteines or the amines on lysines. The conjugation of cytotoxic drugs to the antibodies results in a heterogeneous mixture of ADCs where the number of cytotoxic drugs conjugated to an antibody typically varies from 0 to 8 drugs per antibody. Antibodies with a DAR of 8 or more have a higher clearance rate than antibodies with a lower DAR (11). The majority of the ADCs have an average DAR of approximately 4 drugs per antibody, which offers an acceptable balance between pharmacokinetic properties, toxicities and anti-tumor efficacy.

\section{Site Specific Conjugation}

Cytotoxic drugs are conjugated to amino acids, typically cysteines or non-natural amino acids, which have been incorporated at distinct locations on the antibody. The resulting ADC is homogeneous composition where all of the antibodies contain a defined number of drugs per antibody. Typically a DAR of 2 is used although higher DARs could be achieved. The incorporation of a cytotoxic drug on specific positions of the antibody can influence the pharmacokinetic properties of the ADC. Several examples of site specifically conjugated ADCs, using antibodies containing engineered cysteines or the non-natural amino acid, p-acetyl phenyalanine (pAF), have reported improved in vitro and in vivo serum stability, improved pharmacokinetic properties, comparable antitumor efficacy and improved toxicology profiles as compared to the conventional ADCs $(55,66,67)$. Cytotoxic drugs conjugated to $\mathrm{pAF}$ via an oxime bond are reported to have improved serum stability when compared to cytotoxic drugs conjugated to thiols via maleimide $(10,55)$.

One cysteine engineered site specifically conjugated ADC, SGN-GD33A, is currently in phase I clinical development. This is probably the first of many site specific ADCs that will be evaluated in the clinic.

A couple of novel approaches for site-specific conjugation not requiring molecular engineering of the antibody are cysteine bridging approaches, which can specifically conjugate all of the cysteines with a defined DAR or $1 / 2$ the number of cysteines (4 for a typical IgG1), and conjugation of glycosyl moieties.

There are several companies developing site specific conjugation technologies but most are currently in preclinical development (Table VII). This is an exciting area of development for ADCs. 
Table VII Companies Developing Site Specific ADC Technologies

\begin{tabular}{lll}
\hline Company & Type of Technology & Stage of Development \\
\hline Ambrx Inc & Non-natural amino acid (NNAA) & Preclinical \\
Genentech/Roche, Novartis & Engineered cysteine (Thiomab) & Preclinical \\
Igenica Inc & (SNAP ADC) no mutation or NNAA & Preclinical \\
Meditope Bioscience Inc & Cetuximab derived residue grafts into Fab & Preclinical \\
Pfizer & FXIII substrate peptide - acceptor glutamine & Preclinical \\
Poly Therics Ltd & ThioBridge & Preclinical \\
Redwood Bioscience Inc & Aldehyde tag & Preclinical \\
(acquired by Catalent Pharma Solutions) & & \\
Seattle Genetics & Engineered Cysteine & Clinical \\
Sutro Biopharma Inc & Non-natural amino acid & Preclinical \\
SynAffix & Glycosyl conjugation & Preclinical \\
\hline
\end{tabular}

\section{CHALLENGES OF PRECLINICAL DEVELOPMENT OF ADCS}

The data from clinical evaluation of ADCs has led to significant changes in the preclinical development of ADCs. The current cytotoxic drugs used as ADC payloads are more potent than methotrexate, doxorubicin and vinblastine, which were previously used as ADC payloads. The current sets of linkers, used to conjugate the drugs to the antibodies, are more stable than the previous linkers and the amount of unconjugated antibody has been dramatically reduced. These changes were incorporated into the preclinical development of the two FDA approved ADCs, Kadcyla and Adcetris.

The clinical development of more than 15 ADCs has been discontinued because the ADCs did not provide sufficient anti-tumor responses, had unacceptable toxicology profiles or the company decided to support other products in their pipeline. These ADCs apparently had an acceptable preclinical data package that supported the decision to progress them into clinical development. Inotuzumab Ozogamicin (CMC544) has an active Phase III clinical effort and is currently the most advanced ADC in clinical development. CMC-544 showed significant inhibition of tumor growth in various preclinical human B-cell lymphoma cell line models (68). Pfizer, which is developing CMC-544, recently announced that it is discontinuing a Phase III trial in NHL but will continue the Phase III INO-VATE ALL study (B1931022) in ALL. This suggests that preclinical in vivo anti-tumor efficacy studies may not predict patient responses to the ADCs and additional preclinical studies may be warranted to increase the correlation between the data from the preclinical studies and clinical responses.

An area of focus in the preclinical development of ADCs could be in improving the tumor models used to evaluate the ADCs. Preclinical studies have suggested that human tumor xenografts, derived from cell lines, do not accurately reflect the original patient tumors they were derived from and may not predict clinical anti-tumor activity $(69,70)$. Patient derived tumor xenografts (PDX) are derived from freshly isolated patient tumors that are implanted, grown and propagated in immunodeficient mice. A large panel of patient derived colon tumors was reported to accurately represent the histology and the range of molecular heterogeneity observed in colon cancer patients (71). The authors conducted an evaluation of cetuximab in the colorectal cancer PDX tumors and confirmed the key role of KRAS mutation in cetuximab resistance. The increased use of PDX models may provide better predictive value than the use of human tumor cell lines. ADCs against $5 \mathrm{~T} 4$ and tissue factor have reported preclinical in viwo anti-tumor activity in PDX models (72,73).

Perhaps attention to the pharmacokinetics and pharmacodynamics of the ADC may add value to the preclinical effort. An evaluation of a PK/PD modeling approach reported that tumor growth rate and the ratio between the exposures and concentrations that induced tumor stasis were important in predicting tumor responses to Kadcyla (74). This PK/PD model was modified using the clinical PK data for Kadcyla and was used to predict the clinically efficacious exposures for an ADC against the 5T4 tumor antigen (75). The utility of this modeling approach is currently being evaluated.

The use of site specific ADCs, improvements in the pharmacokinetic properties of the ADCs through the development of better linkers and the use of potent cytotoxic drugs as ADC payloads combined with improvements in tumor model selection and target identification may result in higher success rates for ADCs in the clinic.

Open Access This article is distributed under the terms of the Creative Commons Attribution License which permits any use, distribution, and reproduction in any medium, provided the original author(s) and the source are credited. 


\section{REFERENCES}

1. Varki NM, Reisfeld RA, Walker LE. Antigens associated with a human lung adenocarcinoma defined by monoclonal antibodies. Cancer Res. 1984;44:681-7.

2. Schneck D, Butler F, Dugan W, Littrell D, Petersen B, Bowsher R, et al. Disposition of a murine monoclonal antibody vinca conjugate (KS1/4-DAVLB) in patients with adenocarcinomas. Clin Pharmacol Ther. 1990;47:36-41.

3. Elias DJ, Hirschowitz L, Kline LE, Kroener JF, Dillman RO, Walker LE, et al. Phase I clinical comparative study of monoclonal antibody $\mathrm{KSl} / 4$ and KSl/4-methotrexate immunconjugate in patients with non-small cell lung carcinoma. Cancer Res. 1990;50:4154-9.

4. Goldmacher VS, Kovtun YV. Antibody-drug conjugates: using monoclonal antibodies for delivery of cytotoxic payloads to cancer cells. Ther Deliv. 2011;2:397-416.

5. Trail PA, Willner D, Lasch SJ, Henderson AJ, Hofstead S, Casazza AM, et al. Cure of xenografted human carcinomas by BR96doxorubicin immunoconjugates. Science. 1993;261:212-5.

6. Willner D, Trail PA, Hofstead SJ, King HD, Lasch SJ, Braslawsky GR, et al. (6-Maleimidocaproyl)hydrazone of doxorubicin-a new derivative for the preparation of immunoconjugates of doxorubicin. Bioconjug Chem. 1993;4:521-7.

7. Morell A, Terry WD, Waldmann TA. Metabolic properties of IgG subclasses in man. J Clin Invest. 1970;49:673-80.

8. Senter PD, Sievers EL. The discovery and development of brentuximab vedotin for use in relapsed Hodgkin lymphoma and systemic anaplastic large cell lymphoma. Nat Biotechnol. 2012;30:631-7.

9. Senter PD. Potent antibody drug conjugates for cancer therapy. Curr Opin Chem Biol. 2009;13:235-44.

10. Tian F, Lu Y, Manibusan A, Sellers A, Tran H, Sun Y, et al. A general approach to site-specific antibody drug conjugates. Proc Natl Acad Sci U S A. 2014;111:1766-71.

11. Hamblett KJ, Senter PD, Chace DF, Sun MM, Lenox J, Cerveny $\mathrm{CG}$, et al. Effects of drug loading on the antitumor activity of a monoclonal antibody drug conjugate. Clin Cancer Res. 2004;10: 7063-70.

12. Wakankar A, Chen Y, Gokarn Y, Jacobson FS. Analytical methods for physicochemical characterization of antibody drug conjugates. MAbs. 2011;3:161-72.

13. Golay J, Di Gaetano N, Amico D, Cittera E, Barbui AM, Giavazzi R, et al. Gemtuzumab ozogamicin (Mylotarg) has therapeutic activity against CD33 acute lymphoblastic leukaemias in vitro and in vivo. $\mathrm{Br}$ J Haematol. 2005;128:310-7.

14. Ricart AD. Antibody-drug conjugates of calicheamicin derivative: gemtuzumab ozogamicin and inotuzumab ozogamicin. Clin Cancer Res. 2011;17:6417-27.

15. Doronina SO, Toki BE, Torgov MY, Mendelsohn BA, Cerveny CG, Chace DF, et al. Development of potent monoclonal antibody auristatin conjugates for cancer therapy. Nat Biotechnol. 2003;21: 778-84.

16. Widdison WC, Wilhelm SD, Cavanagh EE, Whiteman KR, Leece BA, Kovtun Y, et al. Semisynthetic maytansine analogues for the targeted treatment of cancer. J Med Chem. 2006;49:4392-408.

17. Oroudjev E, Lopus M, Wilson L, Audette C, Provenzano C, Erickson $\mathrm{H}$, et al. Maytansinoid-antibody conjugates induce mitotic arrest by suppressing microtubule dynamic instability. Mol Cancer Ther. 2010;9:2700-13.

18. Griffin JD, Linch D, Sabbath K, Larcom P, Schlossman SF. A monoclonal antibody reactive with normal and leukemic human myeloid progenitor cells. Leuk Res. 1984;8:521-34.

19. Dinndorf PA, Andrews RG, Benjamin D, Ridgway D, Wolff L, Bernstein ID. Expression of normal myeloid-associated antigens by acute leukemia cells. Blood. 1986;67:1048-53.
20. Mejstrikova E, Kalina T, Trka J, Stary J, Hrusak O. Correlation of CD33 with poorer prognosis in childhood ALL implicates a potential of anti-CD33 frontline therapy. Leukemia. 2005;19:1092-4.

21. Jilani I, Estey E, Huh Y, Joe Y, Manshouri T, Yared M, et al. Differences in CD33 intensity between various myeloid neoplasms. Am J Clin Pathol. 2002;1 18:560-6.

22. Lapusan S, Vidriales MB, Thomas X, de Botton S, Vekhoff A, Tang R, et al. Phase I studies of AVE9633, an anti-CD33 antibody-maytansinoid conjugate, in adult patients with relapsed/refractory acute myeloid leukemia. Invest New Drugs. 2012;30:1121-31.

23. Tang R, Cohen S, Perrot JY, Faussat AM, Zuany-Amorim C, Marjanovic $\mathrm{Z}$, et al. $\mathrm{P}$-gp activity is a critical resistance factor against AVE9633 and DM4 cytotoxicity in leukaemia cell lines, but not a major mechanism of chemoresistance in cells from acute myeloid leukaemia patients. BMC Cancer. 2009;9:199.

24. Younes A, Yasothan U, Kirkpatrick P. Brentuximab vedotin. Nat Rev Drug Discov. 2012;11:19-20.

25. Deng C, Pan B, O’Connor OA. Brentuximab vedotin. Clin Cancer Res. 2013;19:22-7.

26. Vaklavas C, Forero-Torres A. Safety and efficacy of brentuximab vedotin in patients with Hodgkin lymphoma or systemic anaplastic large cell lymphoma. Ther Adv Hematol. 2012;3:209-25.

27. Katz J, Janik JE, Younes A. Brentuximab Vedotin (SGN-35). Clin Cancer Res. 201 1;17:6428-36.

28. Garnock-Jones KP. Brentuximab vedotin: a review of its use in patients with hodgkin lymphoma and systemic anaplastic large cell lymphoma following previous treatment failure. Drugs. 2013;73: 371-81.

29. Bartlett NL, Sharman JP, Oki Y, Advani RH, Bello CM, Winter JN, et al. A phase 2 study of brentuximab vedotin in patients with relapsed or refractory CD30-positive non-hodgkin lymphomas: interim results in patients with DLBCL and other B-cell lymphomas. 2013.

30. Yonemura Y, Ninomiya I, Yamaguchi A, Fushida S, Kimura H, Ohoyama S, et al. Evaluation of immunoreactivity for erbB-2 protein as a marker of poor short term prognosis in gastric cancer. Cancer Res. 1991;51:1034-8.

31. Sasano H, Date F, Imatani A, Asaki S, Nagura H. Double immunostaining for c-erbB-2 and p53 in human stomach cancer cells. Hum Pathol. 1993;24:584-9.

32. Uchino S, Tsuda H, Maruyama K, Kinoshita T, Sasako M, Saito $\mathrm{T}$, et al. Overexpression of c-erbB-2 protein in gastric cancer. Its correlation with long-term survival of patients. Cancer. 1993;72: 3179-84.

33. Nakajima M, Sawada H, Yamada Y, Watanabe A, Tatsumi M, Yamashita J, et al. The prognostic significance of amplification and overexpression of c-met and c-erb B-2 in human gastric carcinomas. Cancer. 1999;85:1894-902.

34. Press MF, Cordon-Cardo C, Slamon DJ. Expression of the HER-2/ neu proto-oncogene in normal human adult and fetal tissues. Oncogene. 1990;5:953-62.

35. Verma S, Miles D, Gianni L, Krop IE, Welslau M, Baselga J, et al. Trastuzumab emtansine for HER2-positive advanced breast cancer. N Engl J Med. 2012;367:1783-91.

36. Milosevic MF, Fyles AW, Hill RP. The relationship between elevated interstitial fluid pressure and blood flow in tumors: a bioengineering analysis. Int J Radiat Oncol Biol Phys. 1999;43:1111-23.

37. Dudley AC. Tumor endothelial cells. Cold Spring Harb Perspect Med. 2012;2, a006536.

38. Choi IK, Strauss R, Richter M, Yun CO, Lieber A. Strategies to increase drug penetration in solid tumors. Front Oncol. 2013;3:193.

39. Thurber GM, Schmidt MM, Wittrup KD. Antibody tumor penetration: transport opposed by systemic and antigen-mediated clearance. Adv Drug Deliv Rev. 2008;60:1421-34. 
40. Fujimori K, Covell DG, Fletcher JE, Weinstein JN. A modeling analysis of monoclonal antibody percolation through tumors: a binding-site barrier. J Nucl Med. 1990;31:1191-8.

41. Adams GP, Schier R, McGall AM, Simmons HH, Horak EM, Alpaugh RK, et al. High affinity restricts the localization and tumor penetration of single-chain fv antibody molecules. Cancer Res. 2001:61:4750-5.

42. Sharkey RM, Govindan SV, Cardillo TM, Goldenberg DM. Epratuzumab-SN-38: a new antibody-drug conjugate for the therapy of hematologic malignancies. Mol Cancer Ther. 2012;11:224-34.

43. McDonagh CF, Kim KM, Turcott E, Brown LL, Westendorf L, Feist $\mathrm{T}$, et al. Engineered anti-CD70 antibody-drug conjugate with increased therapeutic index. Mol Cancer Ther. 2008;7:2913-23.

44. Wypych J, Li M, Guo A, Zhang Z, Martinez T, Allen MJ, et al. Human IgG2 antibodies display disulfide-mediated structural isoforms. J Biol Chem. 2008;283:16194-205.

45. Moore GL, Chen H, Karki S, Lazar GA. Engineered Fc variant antibodies with enhanced ability to recruit complement and mediate effector functions. MAbs. 2010;2:181-9.

46. Presta LG. Engineering antibodies for therapy. Curr Pharm Biotechnol. 2002;3:237-56

47. Petricevic B, Laengle J, Singer J, Sachet M, Fazekas J, Steger G, et al. Trastuzumab mediates antibody-dependent cell-mediated cytotoxicity and phagocytosis to the same extent in both adjuvant and metastatic HER2/neu breast cancer patients. J Transl Med. 2013;1 1:307.

48. Ducry L, Stump B. Antibody-drug conjugates: linking cytotoxic payloads to monoclonal antibodies. Bioconjug Chem. 2010;21:5-13.

49. Pietersz GA, Rowland A, Smyth MJ, McKenzie IF. Chemoimmunoconjugates for the treatment of cancer. Adv Immunol. 1994;56:301-87.

50. Tolcher AW, Sugarman S, Gelmon KA, Gohen R, Saleh M, Isaacs $\mathrm{C}$, et al. Randomized phase II study of BR96-doxorubicin conjugate in patients with metastatic breast cancer. J Clin Oncol. 1999;17:47884.

51. Tassone P, Gozzini A, Goldmacher V, Shammas MA, Whiteman $\mathrm{KR}$, Carrasco DR, et al. In vitro and in vivo activity of the maytansinoid immunoconjugate huN901-N2'-deacetyl-N2'-(3mercapto-1-oxopropyl)-maytansine against CD56+ multiple myeloma cells. Cancer Res. 2004;64:4629-36.

52. Okeley NM, Miyamoto JB, Zhang X, Sanderson RJ, Benjamin DR, Sievers EL, et al. Intracellular activation of SGN-35, a potent antiCD30 antibody-drug conjugate. Clin Cancer Res. 2010;16:888-97.

53. Golfier S, Kopitz C, Kahnert A, Heisler I, Schatz CA, Stelte-Ludwig $\mathrm{B}$, et al. Anetumab ravtansine: a novel mesothelin-targeting antibodydrug conjugate cures tumors with heterogeneous target expression favored by bystander effect. Mol Cancer Ther. 2014;13:1537-48.

54. Alley SC, Benjamin DR, Jeffrey SC, Okeley NM, Meyer DL, Sanderson RJ, et al. Contribution of linker stability to the activities of anticancer immunoconjugates. Bioconjug Chem. 2008;19:759-65.

55. Jackson D, Atkinson J, Guevara CI, Zhang C, Kery V, Moon $\mathrm{SJ}$, et al. In vitro and in vivo evaluation of cysteine and site specific conjugated herceptin antibody-drug conjugates. PLoS One. 2014;9:e83865.

56. Tolcher AW, Ochoa L, Hammond LA, Patnaik A, Edwards T, Takimoto $\mathrm{C}$, et al. Cantuzumab mertansine, a maytansinoid immunoconjugate directed to the CanAg antigen: a phase I, pharmacokinetic, and biologic correlative study. J Clin Oncol. 2003;21: 211-22.

57. Burris 3rd HA, Rugo HS, Vukelja SJ, Vogel CL, Borson RA, Limentani S, et al. Phase II study of the antibody drug conjugate
trastuzumab-DM1 for the treatment of human epidermal growth factor receptor 2 (HER2)-positive breast cancer after prior HER2directed therapy. J Clin Oncol. 2011;29:398-405.

58. Lippert TH, Ruoff HJ, Volm M. Intrinsic and acquired drug resistance in malignant tumors. The main reason for therapeutic failure. Arzneimittelforschung. 2008;58:261-4.

59. Gottesman MM. Mechanisms of cancer drug resistance. Annu Rev Med. 2002;53:615-27.

60. Sammet B, Steinkuhler C, Sewald N. Antibody-drug conjugates in tumor therapy. Pharm Pat Anal. 2012;1:65-73.

61. Sievers EL, Senter PD. Antibody-drug conjugates in cancer therapy. Annu Rev Med. 2013;64:15-29.

62. Loo TW, Clarke DM. Recent progress in understanding the mechanism of P-glycoprotein-mediated drug efflux. J Membr Biol. 2005;206:173-85.

63. Kovtun YV, Audette CA, Mayo MF, Jones GE, Doherty H, Maloney EK, et al. Antibody-maytansinoid conjugates designed to bypass multidrug resistance. Cancer Res. 2010;70:2528-37.

64. Lebedeva IV, Pande P, Patton WF. Sensitive and specific fluorescent probes for functional analysis of the three major types of mammalian ABC transporters. PLoS One. 201 1;6:e22429.

65. Forster S, Thumser AE, Hood SR, Plant N. Characterization of rhodamine-123 as a tracer dye for use in in vitro drug transport assays. PLoS One. 2012;7:e33253.

66. Junutula JR, Raab H, Clark S, Bhakta S, Leipold DD, Weir S, et al. Site-specific conjugation of a cytotoxic drug to an antibody improves the therapeutic index. Nat Biotechnol. 2008;26:925-32.

67. Junutula JR, Flagella KM, Graham RA, Parsons KL, Ha E, Raab H, et al. Engineered thio-trastuzumab-DMl conjugate with an improved therapeutic index to target human epidermal growth factor receptor 2-positive breast cancer. Clin Cancer Res. 2010;16:4769-78.

68. DiJoseph JF, Armellino DG, Boghaert ER, Khandke K, Dougher MM, Sridharan L, et al. Antibody-targeted chemotherapy with CMC-544: a CD22-targeted immunoconjugate of calicheamicin for the treatment of B-lymphoid malignancies. Blood. 2004;103: 1807-14.

69. Siolas D, Hannon GJ. Patient-derived tumor xenografts: transforming clinical samples into mouse models. Cancer Res. 2013;73:5315-9.

70. Sausville EA, Burger AM. Contributions of human tumor xenografts to anticancer drug development. Cancer Res. 2006;66:3351-4. discussion 3354

71. Julien S, Merino-Trigo A, Lacroix L, Pocard M, Goere D, Mariani $\mathrm{P}$, et al. Characterization of a large panel of patient-derived tumor xenografts representing the clinical heterogeneity of human colorectal cancer. Clin Cancer Res. 2012;18:5314-28.

72. Breij EC, de Goeij BE, Verploegen S, Schuurhuis DH, Amirkhosravi A, Francis J, et al. An antibody-drug conjugate that targets tissue factor exhibits potent therapeutic activity against a broad range of solid tumors. Cancer Res. 2014;74:1214-26.

73. Sapra P, Damelin M, Dijoseph J, Marquette K, Geles KG, Golas J, et al. Long-term tumor regression induced by an antibody-drug conjugate that targets 5T4, an oncofetal antigen expressed on tumor-initiating cells. Mol Cancer Ther. 2013;12:38-47.

74. Jumbe NL, Xin Y, Leipold DD, Crocker L, Dugger D, Mai E, et al. Modeling the efficacy of trastuzumab-DM1, an antibody drug conjugate, in mice. J Pharmacokinet Pharmacodyn. 2010;37:221-42.

75. Haddish-Berhane N, Shah DK, Ma D, Leal M, Gerber HP, Sapra P, et al. On translation of antibody drug conjugates efficacy from mouse experimental tumors to the clinic: a PK/PD approach. J Pharmacokinet Pharmacodyn. 2013;40:557-71. 